\title{
Multiple Cell Partitions for Increasing the CDMA-Based Cell Capacity
}

\author{
Ardian Ulvan $^{1}$, Diogo Ribeiro ${ }^{2}$ and Robert Bestak ${ }^{1}$ \\ ${ }^{1}$ Czech Technical University in Prague, Technicka 2166 27, Praha 6, Czech Republic \\ ulvana1, bestar1[@fel.cvut.cz] \\ ${ }^{2}$ Instituto Superior Técnico de Lisboa, Av. Rovisco Pais, 1049-001 Lisboa, Portugal \\ dpbr@mega.ist.utl.pt
}

\begin{abstract}
This paper investigates the impact of cell partitioning in cell capacity of CDMA-based system. We examine the implementation of four partitions per cell as well as the influence of various height of the base station transceiver antenna to the system. We contemplated the urban area as the target, therefore the COST-231 Walfisch - Ikegami is applied as the propagation model. Calculations on the capacity of cell, the link budget (and MAPL) and the radius of each partition, depending on the height of the base station transceiver antenna, are made. The results show that the capacity increases as the number of partitions increases; the height of the base station transceiver affects the radius of the partitions.
\end{abstract}

\section{Introduction}

The conventional method for cellular planning is based on multi-cell configuration. The topology has a base transceiver station (BTS) that serves areas divided in cells or several BTS covering the whole area. However, in this method, to avoid the co-channel interference, each adjacent cell needs to use different frequencies. In practice, the spacing has to be more than just adjacent cells, around 7 frequencies are often used per planning. Thus, to increase the capacity, the number of frequency channels would have to be increased. Since frequency is a precious resource and in most cities the growth of traffic tends to concentrate in certain area only, this method becomes very inefficient.

The use of CDMA-based systems in this work is due to its potential concerning capacity increase and frequency spectrum management. However, since this type of systems are strongly influenced by interference between users or even the one that comes from base transceiver stations, the way to improve capacity is to control this sources of interference. These two types are called inter-user interference and co-channel interference. This paper describes the use of cell partition as an alternative solution for CDMA-based cellular planning.

In this paper we analyze the effect of increasing the number of partitions and varying the BTS's antenna height. 
Resorting to a variable number of partitions, an existing cell is divided into 4 partitions, being scaled in radius as well as in frequency. In order to determine the radius of each partition, it's required the calculation of link budget, which provides the maximum allowable path loss (MAPL) and the usage of a propagation model, which is in this case the Walfisch - Ikegami. After having all of this data, it's possible to determine the maximum capacity of the cell.

The paper is organized as follows: the definition of the cell partition for improving cell capacity, frequency planning and channel allocation scheme for partitioned cell, link budget and cell radius calculations are discussed in Section II. Section III proposes three and four partitions topology in each cell that are used in or simulation scenarios. In Section IV we discussed the results of total capacity of the system and compared them with the capacity of conventional frequency planning. Finally, section V and VI concludes the work and assign our future work.

\section{Cell Capacity}

\subsection{Variable number of partitions}

Increasing the cell capacity by using 2-partition topology was carried out by [1]. However, the used of two different frequencies for inner and outer cell seems have low complexity in term of interference. Additionally, the cell radius which is influenced by antenna altitude was not considered yet. This paper intends to determine the complexity by increase the number of partitions and analyze what happens to the total capacity of the cell. A cell is divided into four partitions, all of them with a radius disparity that is scaled between them. The way to perform the scaling of radius is to vary the height of the antennas and the transmitting power of each BTS [5]. So, the scheme of one cell is shown in figure 1.a below.

If this cell is spanned throughout a whole coverage area, the frequencies within each partition have to be alternated, and so, for a 3 and 4 partitions scheme, we have a frequency planning for each partitions as can be seen on figure 1.b.

Note that in this figure each colour corresponds to one different frequency, so the goal of not having boundaries with common frequencies on each side is achieved.

The frequency planning is the key to improve the capacity of the system. Each partition within the cell, have a different frequency not to cause interference between the partitions. It means that with three partitions we have a frequency reuse factor of three, with four partitions, a reuse factor of four, and so on. To maximize the capacity of each cell, one has to minimize the interference, since CDMA is an interference limited technology. When a mobile station moves to the cell's boundary, the interference from neighboring cells increase considerably. The worst case for the forward link occurs at the boundary of three cells where powers received from two adjacent cells are almost equal to that from the given cell. Taking this fact into consideration, the goal of the frequency planning is to 
avoid the existence of cell boundaries with the same frequency in each of the outer partitions that compose this boundary, which yield the distribution in figure 1.b for a 3 cell partition scheme, and in figure 1.c for a 4 cell partition topology.

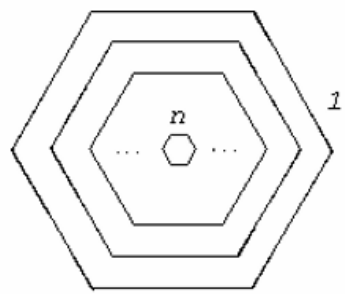

a.

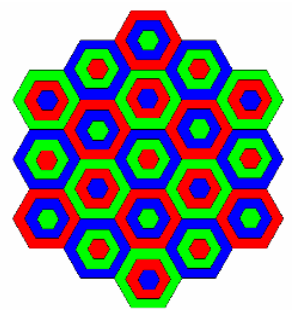

b.

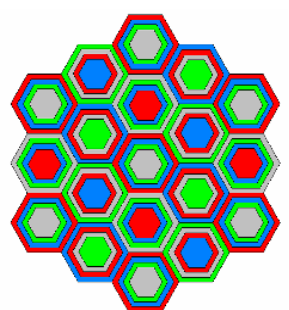

c.

Fig.1. Schematic representation of a cell containing $n$ partitions (a) and frequency planning for a scheme with 3 and 4 partitions per cell $(b, c)$

Accordingly as it is mentioned, each partition is designed to use different frequencies. The outer most partition always includes the coverage of the other ones, consequently there are as many frequencies as the number of partitions in the outer most cell. However, there is no interference since all of the frequencies are different.

Concerning the traffic allocation process it becomes slightly different from the one without partitions. The traffic allocation process is always prioritised to the inner most cell. If a mobile station (MS) is in the inner most cell coverage, the allocation of channel is served by its frequency and the connection is held by the inner most cell's radio base station (RBS). On the other hand, if there are no idle channels in the inner most cells, the connection is relocated to the next partition (the second smallest in radius) and the system tries to establish the connection here. If it is not possible as well, the system tries the next partitions in an orderly fashion from the smallest to the biggest radius partition, until the connection is established or rejected. The rejection can be due to all of the radio base stations not having idle traffic channels or the mobile station moved farther away than the radius of the outer most cells. When the connection is established, it is served by the frequency of the partition where an idle channel has been found. In figure 2, it is shown the algorithm of traffic channel allocation in a cell with an indefinite number of partitions. 


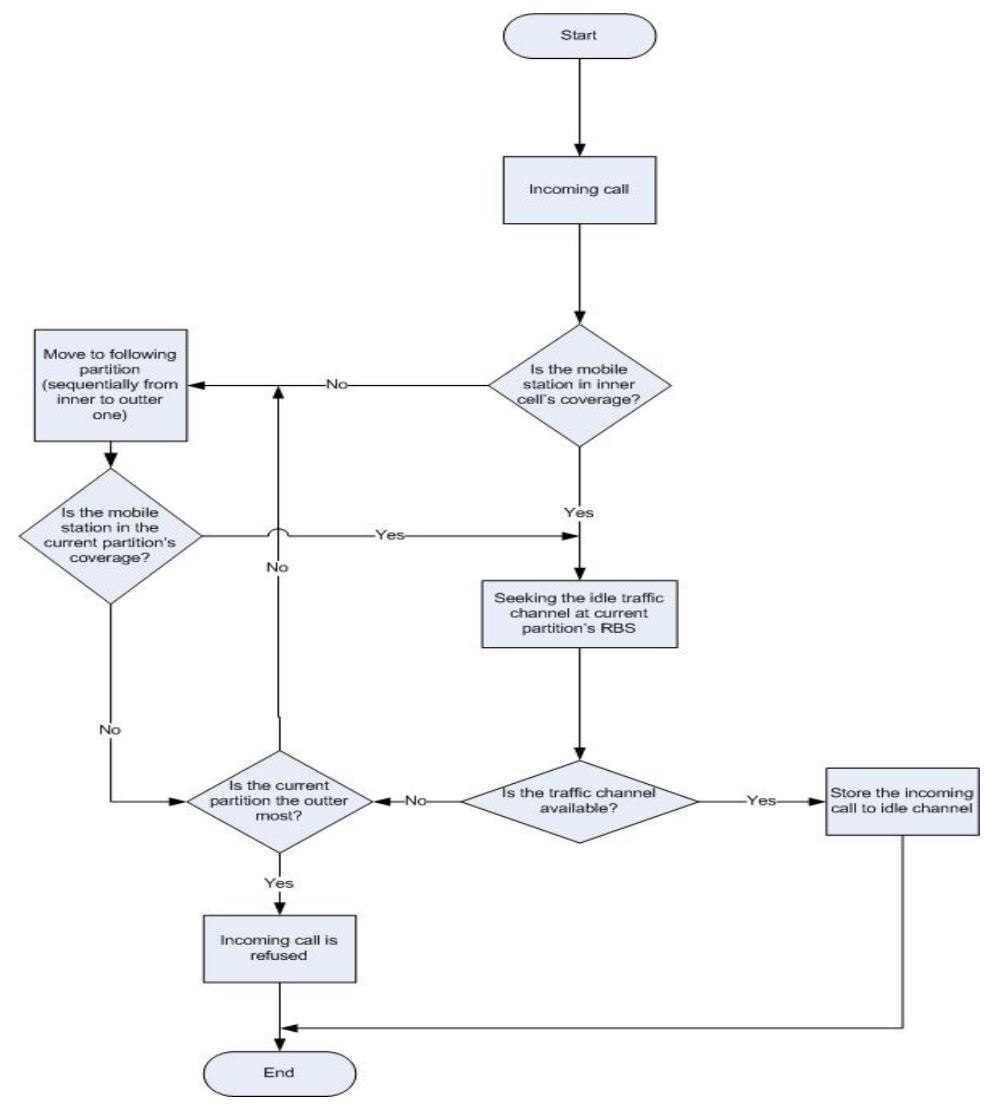

Fig. 2. Channel allocation algorithm for an indefinite number of partitions

\subsection{CDMA Considerations}

In CDMA systems the $\mathrm{W}^{64}$ of Walsh code generates 1 pilot channel, 1 synchronization channel, 7 paging channels and 55 traffic channels. To allow enough bandwidth for data and for bandguard, the 55 traffic channels can be served by $1.25 \mathrm{MHz}$ of bandwidth. The capacity of the system, which is represented by the number of users that the system can support, with a single cell topology is [6]:

$$
N=1+\frac{W / R}{E_{b} / N_{0}}-(\eta / S)
$$

In above equation it was considered the background thermal noise, $\eta$, which has to be taken into account in the spread bandwidth. 


\subsection{Link Budget}

To determine the radius, it needs to be known the link budget, this allows the calculation of the total losses and gains on the transmission link. It is known that the signal in receiver can be detected if the following condition is reached [5]:

$$
\begin{aligned}
& \left(P_{t r}\right)_{d B m}+(\text { Gains })_{d B}-(\text { Losses })_{d B}>\text { (Min. required power } \\
& \text { for detection at the receiver })_{\mathrm{dBm}}
\end{aligned}
$$

The result of the link budget calculation is a cell's MAPL (Maximum Allowable Path Loss). This calculation is employed in both directions: the reverse link and the forward link. The MAPL for reverse and forward link is described in the following equations, respectively [2]:

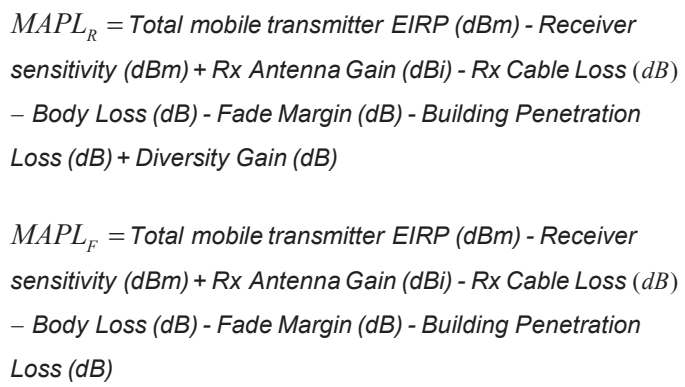

The results attained for the link budget calculations are as follow.

Table 1. Results of Link Budget Calculation for a cell with three partitions.

\begin{tabular}{lcccc}
\hline & Unit & $\begin{array}{c}\text { Outer } \\
\text { Cell }\end{array}$ & $\begin{array}{c}\text { Medium } \\
\text { Cell }\end{array}$ & $\begin{array}{c}\text { Inner } \\
\text { Cell }\end{array}$ \\
\hline $\begin{array}{l}\text { BTS's } \\
\begin{array}{l}\text { Maximum } \\
\text { transmitting } \\
\text { power } \\
\text { MAPL }\end{array}\end{array}$ & $\mathrm{dBm}$ & 35.00 & 30.00 & 25.00 \\
\hline
\end{tabular}


Table 2. Results of Link Budget Calculation for a cell with four partitions

\begin{tabular}{lccccc}
\hline & Unit & $\begin{array}{c}\text { Outer } \\
\text { Cell }\end{array}$ & $\begin{array}{c}2^{\text {nd }} \text { tier } \\
\text { Cell }\end{array}$ & $\begin{array}{c}3^{\text {rd }} \text { tier } \\
\text { Cell }\end{array}$ & $\begin{array}{c}\text { Inner } \\
\text { Cell }\end{array}$ \\
\hline $\begin{array}{l}\text { BTS's } \\
\text { Maximum } \\
\text { transmitting } \\
\text { power } \\
\text { MAPL }\end{array}$ & $\mathrm{dBm}$ & 40.00 & 35.00 & 30.00 & 25.00 \\
\hline
\end{tabular}

\subsection{Cell Partition's radius}

By using the MAPL, it is possible to calculate the radius of a desired cell/partition using propagation models that are adequate for the environment where the cells/partitions are inserted. We consider the COST 231 propagation model, suitable for urban and dense urban areas.

To determine the cell radius, accurate data of the street and building altitude is required. The cell radius is assumed to be less than $5 \mathrm{~km}$ and the antenna elevation less than $70 \mathrm{~m}$ and more than the average building height. The model employed here consists in several equations with restriction and conditions that are to be used combined. So, mathematical descriptions of the model are [3] [4]:

$$
L=\mathrm{L}_{0}+\mathrm{L}_{\mathrm{rts}}+\mathrm{L}_{\mathrm{msd}}
$$

note:

$L=$ path loss $(d B), L_{0}=$ free space loss $(d B), L_{r t s}=$ roof-top-street diffraction and scatter loss, $L_{m s d}=$ multi-screen diffraction loss.

$$
\begin{gathered}
L_{0}=32,4+20 . \log (r)+20 . \log (f) \\
L_{r t s}=-16,9-10 \cdot \log (w)+10 \cdot \log (f)+20 \cdot \log \left(\Delta_{\text {mobile }}\right)+L_{\text {street }} \\
\text { for } \Delta_{\text {mobile }}>0 \\
L_{r t s}=0 \quad \text { for } \Delta_{\text {mobile }} \leq 0 \\
L_{\text {street }}=-10+0,354 \phi \quad \text { for } 0 \leq \phi<35 \\
L_{\text {street }}=2,5+0,075(\phi-35) \quad \text { for } 35 \leq \phi<55
\end{gathered}
$$




$$
L_{\text {street }}=4,0-0,114(\phi-55) \text { for } 55 \leq \phi \leq 90
$$

note:

$h_{\text {roof }}=$ average of roof building altitude $(m), h_{\text {mobile }}=$ antenna altitude for $M S(m), h_{B T S}=$ antenna altitude for BTS $(m), \Delta_{\text {mobile }}=h_{\text {roof }} h_{\text {mobile }}(m), \Delta_{B T S}=h_{B T S} h_{\text {roof }}(m), w=$ average of street width $(m), \phi=$ road orientation concerning to direct radio path (degree).

$$
L_{m s d}=L_{m e d}+k_{a}+k_{r} \log (r)+k_{f} \log (f)-9 \log (b)
$$

where:

$$
\begin{gathered}
L_{m e d}=-18 \log \left(1+\Delta_{B T S}\right) \text { for } \Delta_{B T S}>0 \\
L_{m e d}=0 \quad \text { for } \Delta_{B T S} \leq 0 \\
k_{a}=54 \quad \text { for } \Delta_{\mathrm{BTS}}>0 \\
k_{a}=54-0,8 \Delta_{\mathrm{BTS}} \text { for } r \geq 0,5 \text { and } \Delta_{\mathrm{BTS}} \leq 0 \\
k_{a}=54-1,6 \Delta_{\mathrm{BTS}} r \text { for } r<0,5 \text { and } \Delta_{\mathrm{BTS}} \leq 0 \\
k_{r}=18 \text { for } \Delta_{\mathrm{BTS}}>0 \\
k_{r}=18-15 \frac{\Delta_{\mathrm{BTS}}}{h_{\text {roof }}} \text { for } \Delta_{\mathrm{BTS}} \leq 0 \\
k_{f}=-4+0,7\left(\frac{f}{925}-1\right)
\end{gathered}
$$

for urban and suburban area

$$
k_{f}=-4+1,5\left(\frac{f}{925}-1\right)
$$

for dense urban area 
note:

$b$ is the average interbuilding distance $(m), k_{a}$ and $k_{r}$ are the correction constants of antenna altitude, $k f$ is the adaptation constant for diverse building density.

In this paper, the height of the BTS antenna will be varied, so the results for the radiuses will be dependent of it. In figure 3.a and 3.b, the results of the calculations are explicit for a 3 and 4 partitions scheme, respectively:

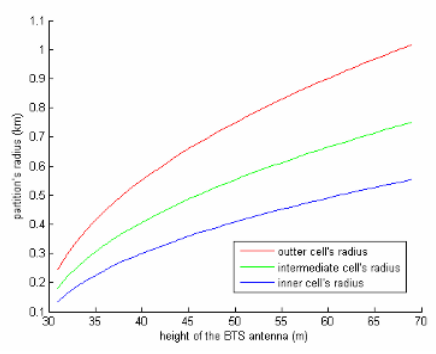

a.

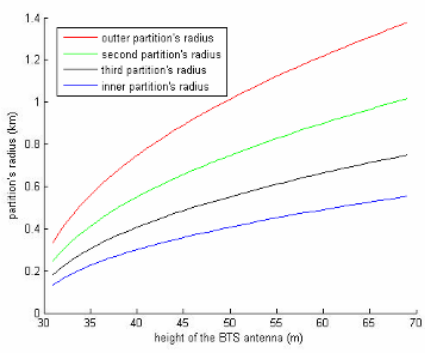

b.

Fig. 3. Variation of the several partitions' radiuses with the height of the BTS antenna for 3 (a) and 4-partitions topology (b).

\section{Cell Capacity with 3 and 4 Partitions Topology}

The equation (1) allows the calculation for the capacity in a single-cell scheme. With a multipartitional topology per cell some other considerations have to be taken into account. However, when the cell partition is done, a different frequency is used for each of the resulting cells and since they do not interfere with one another, it's possible to calculate the capacity individually for each of these. Afterwards, all of the capacities are summed to yield the final result. So, the total capacity of the cell with the partitions is be given by:

$$
N_{\text {total }}=\sum_{i=1}^{n} N_{i}
$$

where $\mathrm{N}_{\text {total }}$ is the total capacity of the cell with the partitions and $\mathrm{N}_{\mathrm{i}}$ is the capacity of the $i^{\text {th }}$ partition. Due to this separation principle, for an arbitrary partition of the cell, the $\mathrm{S} / \mathrm{I}$ ratio is given as: 


$$
S N R=\frac{P_{t} \cdot(1 / N) \cdot r^{-\alpha}}{P_{t} \cdot\left(1-\frac{1}{N}\right) \cdot r^{-\alpha}+\sum_{k=1}^{3} \sum_{j=1}^{6} P_{k j} \cdot L_{k j}^{-\alpha}}
$$

then

$$
N_{i}=\frac{P_{i} \cdot r_{i}^{-\alpha} \cdot\left(1+\frac{S}{I}\right)}{\frac{S}{I} \cdot\left(P_{i} \cdot r_{i}^{-\alpha}+\sum_{k=1}^{3} \sum_{j=1}^{6} P_{k j} L_{k j}^{-\alpha}\right)}
$$

where

$$
\frac{S}{I}=\frac{E_{b} / I_{0}}{W / R}=\frac{E_{b} / I_{0}}{G_{P}}
$$

In the above equations $\mathrm{S} / \mathrm{I}$ is the signal to interference ratio, $\mathrm{P}_{\mathrm{i}}$ is the transmitted EIRP of the partition (Watt), $r_{i}$ is the radius of the partition $(\mathrm{km}), \mathrm{P}_{\mathrm{kj}}$ is the transmitted power of neighbour cells (Watt), $\mathrm{L}_{\mathrm{kj}}$ is the distance between neighbour base station to MS (km), $\alpha$ is the path loss exponent and $\mathrm{N}_{\mathrm{i}}$ is the $i{ }^{\text {th }}$ partition capacity (number of users).

\section{Results for the Traffic Capacity}

Using all of the mentioned equations, the results from the link budget calculation and the radiuses for each partition, are dependent of the height of each BTS. It is now possible to determine the total capacity of the system. This capacity is calculated for 3 and 4 partitions and depends on the BTS's antenna height. Figures 4.a and 4.b show the results of the capacity per partition per cell. In figure 4.c we can see the total capacity of the resulting cell. Tables 3 and 4 are the results for the capacity attained when using the conventional method and 2 partitions per cell topology. 


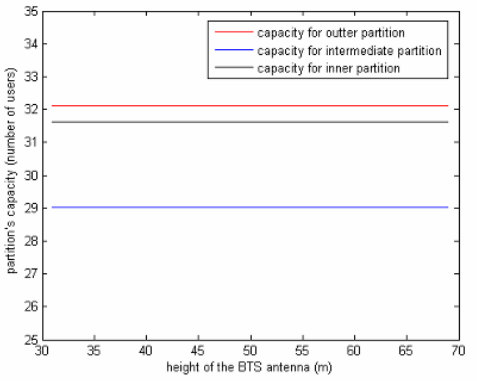

a.

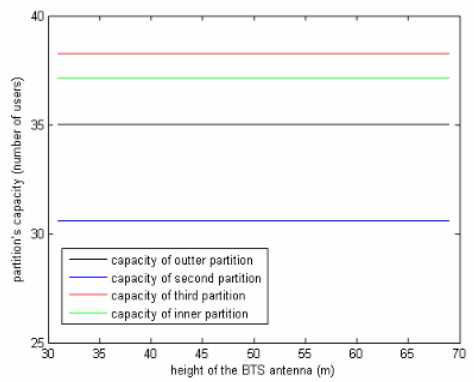

b.

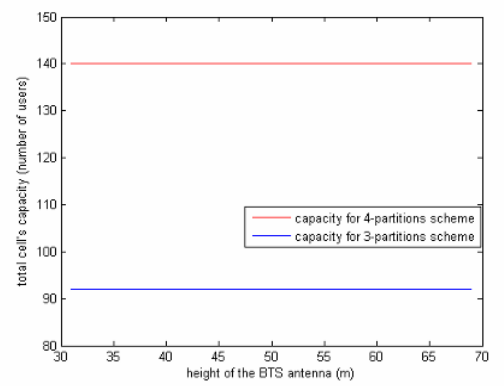

c.

Fig. 4. Capacity of each partition depending on the height of the BTS antenna for 3partitions scheme (a), 4-partitions scheme (b) and total cell's capacity for a topology with 3 and 4 partitions per cell.

Table 3. Results for calculation and simulation of capacity using conventional method (2 frequencies).

\begin{tabular}{clcc}
\hline & Unit & RBS 1 & RBS 2 \\
\hline BTS's EIRP & $\mathrm{dBm}$ & 45 & 45 \\
Frequency & $\mathrm{MHz}$ & 1967.50 & 1966.25 \\
Antenna altitude & $\mathrm{m}$ & 50 & 50 \\
MAPL & $\mathrm{dBm}$ & 130.8 & 130.8 \\
Cell radius & $\mathrm{Km}$ & 0.75 & 0.75 \\
Traffic capacity & users & 29 & 29 \\
\hline
\end{tabular}


Table 4. Results for calculation and simulation of capacity using a topology with 2 partitions per cell.

\begin{tabular}{cccc}
\hline & Unit & Inner cell & Outer cell \\
\hline BTS's EIRP & $\mathrm{dBm}$ & 34 & 45 \\
Frequency & $\mathrm{MHz}$ & 1967.50 & 1966.25 \\
Antenna altitude & $\mathrm{m}$ & 40 & 50 \\
MAPL & $\mathrm{dBm}$ & 123.8 & 130.8 \\
$\begin{array}{c}\text { Cell radius } \\
\text { Traffic }\end{array}$ & $\mathrm{Km}$ & 0.36 & 0.75 \\
capacity & users & 45 & 35 \\
\hline
\end{tabular}

\section{Conclusions}

Obtained results show that the cell capacity can be increased by using cell partitions. Furthermore, the capacity is increased linearly with the number of partitions used per cell. In case of 3 partitions the total cell capacity is increased to 92 users; and in case of 4 partitions per cell it is increased to 140 users. Using the conventional method, the capacity is only 58 users per cell and with 2 partitions per cell 80 users. The CDMA-based systems are indeed interference dependent. It implies that the only parameter affecting the capacity is the interference among users or caused by another BTS which is emitting using the same frequency. Concerning the capacity for each partition inside the cell, simulation results show that the inner cell has a very high capacity comparatively to the others.

The increase in the number of partitions has also the great advantage of allowing an effective frequency planning, since more frequencies are allowed to be used, and one can choose in which partition the capacity is to be increased.

\section{Further Work}

As discussed, the increase of the number of partitions increases the capacity in CDMA-based systems. However, increasing the number of partitions brings some drawbacks. The use of $n$-frequencies per cell, corresponding to n-partitions per cell, will lead to hard-handoff when the mobile station is leaving the coverage area from inside to outside of the cell. The hard handoff can lead to a degradation of the quality of service as well as the grade of service, which are two very important parameters that specify the minimum conditions for an acceptable conversation. 


\section{Acknowledgement}

This research work was supported by grant of Czech Ministry of Education, Youth and Sports No. MSM6840770014.

\section{References}

1. Ulvan, A (2006) Increasing the CDMA-based Cell Capacity for Urban Area With Cell Partition. In Research in Telecommunication Technology. Proceeding, Vol.1, pp $176-$ 181. ISBN 80-8070-637-9.

2. Kim, W. S. and Prabhu, V. K (1998) Enhanced Capacity in CDMA System with Alternate Frequency Planning. IEEE International Conference on Communication. Vol. 2, pp 972-978. ISBN 0-7803-4788-9.

3. Hecker, A., Neuland, M. and Kuerner, T. (2006) Propagation models for high sites in urban areas. Advance in Radio Science, Vol 4, pp 345-349.

4. Hecker, A., and Kuerner, T. (2005) Analysis of Propagation Models for UMTS Ultra High Sites in Urban Area. IEEE $16^{\text {th }}$ International Symposium on Personal, Indoor and Mobile Radio Communications (PIMRC), Vol 4, pp 2337 - 2341. ISBN 9783800729098.

5. Rappaport, T. S. (1996) Wireless communications principles and pratice", Prentice Hall PTR.

6. Ericsson Academy (2006) CDMA2000 RF Engineering Workshop. 